\title{
Characteristic Magnetic Resonance Image Features of Acute Network Injury in Young Patients
}

\author{
Sohyeon Kim ${ }^{a}$ Sung-II Sohn ${ }^{a}$ Hyungjong Park ${ }^{a}$ JoonSik Kim ${ }^{b}$ \\ Jeong-Ho Hong ${ }^{\text {a }}$ \\ aDepartment of Neurology, Dongsan Hospital, Keimyung University School of Medicine, \\ Daegu, South Korea; ${ }^{b}$ Department of Pediatrics, Dongsan Hospital, Keimyung University \\ School of Medicine, Daegu, South Korea
}

Keywords

Stroke $\cdot$ Child $\cdot$ Magnetic resonance imaging

\begin{abstract}
Cerebral infarction is known to cause secondary degeneration of the areas connected to the primarily damaged regions. This has been named as acute network injury and is usually recognized in newborns or babies by high signal intensity on diffusion-weighted imaging (DWI). In this article, we present 2 cases demonstrating several characteristics of network injury. Some features are comparable to previous studies and others are distinctive to our cases. The patients not only showed secondary injury in the thorough pyramidal tract along the longitudinal extensions of neural tracts as expected but also followed transverse connections to reach the contralateral hemisphere. The location of network injury varied according to the initial lesion and projected in an omnidirectional manner as long as the brain parts are interconnected. In addition, the cases well demonstrated the temporal changes on brain imaging. Network injury appeared on DWI around a week after major damage and then subsequently disappeared. The overall process of appearance to disappearance was completed within 2 weeks from the symptom onset. As ominous neurological outcomes are thought to be related to acute network injuries, a comprehensive understanding of the phenomenon is pivotal in improving diagnosis and management.
\end{abstract}




\section{Introduction}

Stroke is a major medical concern worldwide. Although old age is known as one of the crucial risk factors of stroke, people of any age can suffer. Currently, cerebral infarction is thought not to be uncommon in children and neonates. The reported incidence varies depending on the studies, with the highest incidence ranging from $1 / 2,300$ to $1 / 5,000$ per live births in neonates [1]. The clinical presentation of pediatric cerebral infarction is often nonspecific. Babies present with seizures, apnea, lethargy, feeding difficulties, or hypotonia and not with focal neurological symptoms [2]. This reality emphasizes the importance of neuroimaging in young patients. Cerebral infarction can cause secondary degeneration of the areas connected to the primarily damaged regions. This has been named as acute network injury and is recognized by a high-intensity signal on diffusion-weighted imaging (DWI). Studies have suggested the corticospinal tract, thalamus, and corpus callosum as the sites of network injury [2,3]. Lesions are mostly found longitudinally along the popular neural tracts. In addition, they gradually disappear on brain imaging with the elapse of time as primary infarction does [4].

Here, we report 2 young patients with cerebral infarction presenting with distinctive image features of acute network injury. The locations and temporal evolutions are well depicted.

\section{Case Presentation}

Case A

A male child was delivered as the second baby of the twin by cesarean section. He was born after 37 weeks of gestation with a body weight of 2,700 g. Gestation and delivery were uneventful. Physical examination revealed no abnormalities at the time. The baby spontaneously developed generalized tonic-clonic seizure at 2 months of age. On neurological examination, he was responsive to stimulus equally on either side and had no focal motor deficit or abnormal reflexes. Electroencephalography showed ictal waves in the right frontal area with focal slowing over the right hemisphere. Brain magnetic resonance imaging (MRI) was performed to determine the cause of seizure on the day of admission and revealed right middle cerebral artery (MCA) territory infarction involving the right frontal lobe (Fig. 1a). Focal narrowing was suspected with post-stenotic dilatation on the right distal MCA. The patient was treated with aspirin and antiepileptic drugs, levetiracetam, and fosphenytoin. Along with the treatment, seizure or any abnormal symptoms did not recur. Follow-up brain MRI was performed on hospital day 7 (Fig. 1b). A decreased extent of cytotoxic and vasogenic edema was seen, and lesions had changed to subacute infarction. Interestingly, the bilateral genu and body of the corpus callosum, right internal capsule, and right dorsomedial nucleus of the thalamus revealed novel high signals in the DWI map.

Case B

A preterm male baby was born at $36+1$ weeks of gestation with a body weight of 2,600 $\mathrm{g}$ (25-50 percentile), height of $48 \mathrm{~cm}$ (50-90 percentile), and head circumference of $32 \mathrm{~cm}$ (10-50 percentile). He was delivered by emergency cesarean section due to mother's preterm labor pain and fever. On delivery, the baby was stained with meconium and had an Apgar score of 4 at $1 \mathrm{~min}$. He was admitted to the neonatal intensive care unit. Brain ultrasound imaging was performed to screen for prematurity on hospital day 6 and showed blurring of the cortical sulci in the right temporal lobe. Brain MRI was performed on day 8 to obtain detailed information about the condition (Fig. 2a). It showed large acute infarction involving

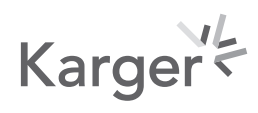




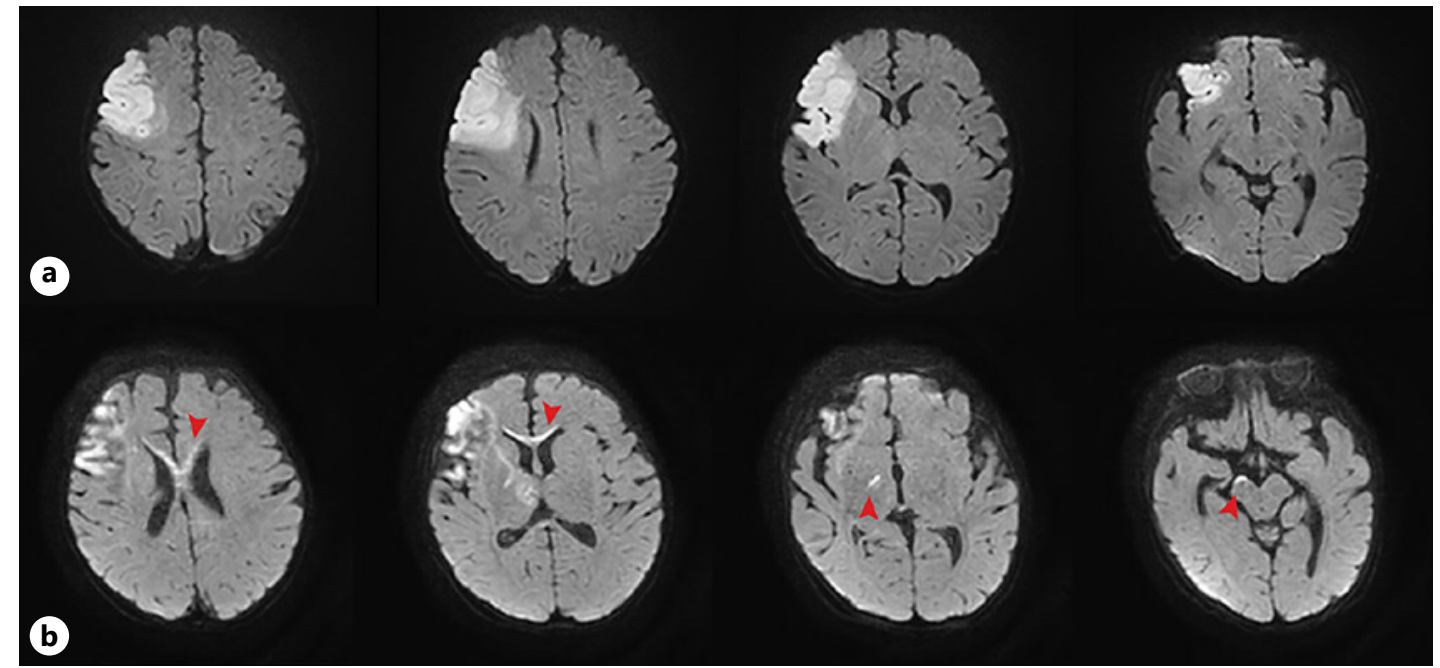

Fig. 1. a Right MCA territory infarction was seen. $\mathbf{b}$ Newly appeared diffusion high signals indicate acute network injury (arrowheads). MCA, middle cerebral artery.

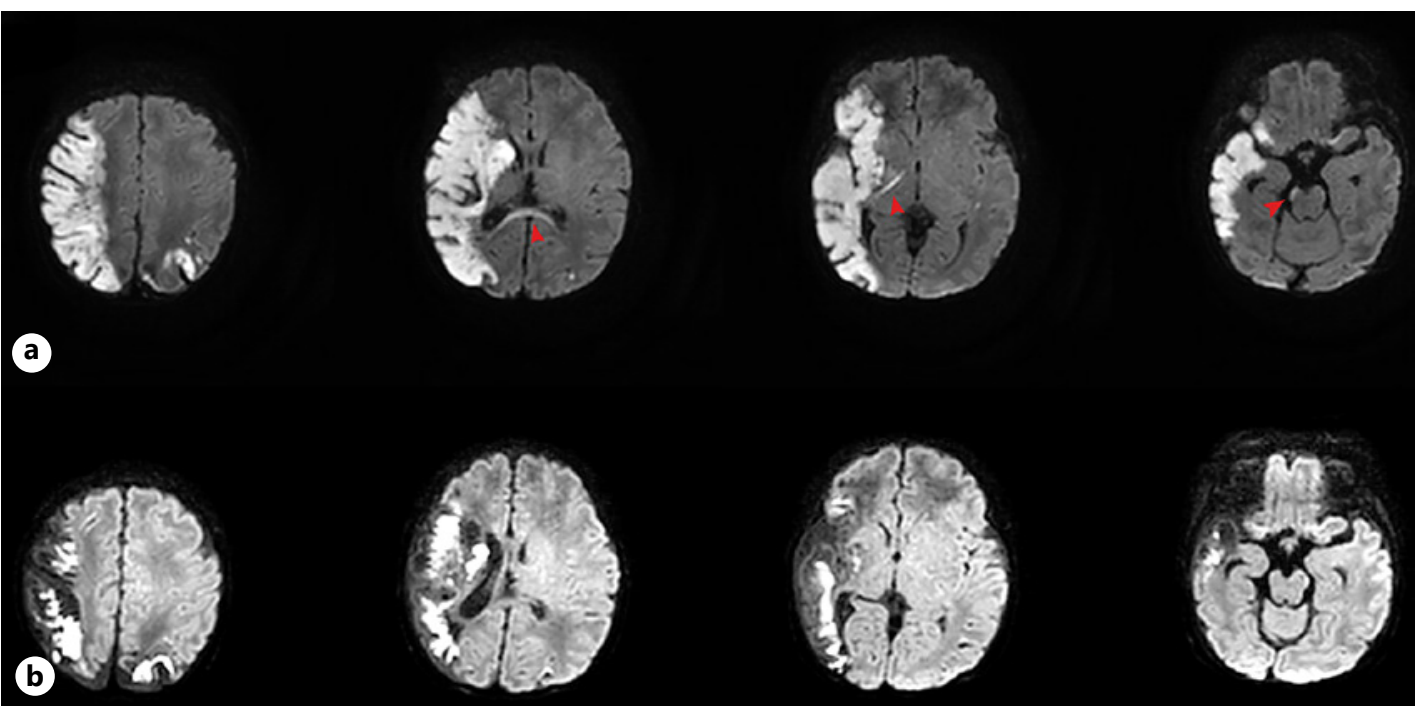

Fig. 2. a High signal intensity in the splenium of corpus callosum, right internal capsule, and right ventral pons indicates that acute network injury had occurred (arrowheads). b Infarcted lesions have undergone maturation, while the signs of acute network injury vanished.

the right MCA and both posterior cerebral artery territories with associated multifocal hemorrhage in the area. DWI high signal intensity was seen in multiple brain lobes as well as in the right posterior limb of the internal capsule and bilateral splenium of the corpus callosum. The patient showed alert mentality with symmetric moro reflex, but the asymmetrical tonic neck reflex seemed uncertain on examination. The baby was treated empirically with an antiepileptic drug. Antithrombotics were not given due to the hemorrhagic lesions. On the follow-up brain MRI on day 15 (Fig. 2b), the infarcted lesions had undergone maturation without any new lesion. In the meanwhile, diffusion high signals of internal capsule and splenium vanished. After discharge, the baby consistently showed focal spikes in the right hemisphere in serial electroencephalography tests, despite the absence of a clinical seizure event. The parents 
reported that mild left arm weakness and left leg spasticity had been presented as he grew up. Antiepileptics were continued, and the patient consulted a physiatrist for rehabilitation of left limb movements.

\section{Discussion}

These 2 cases show several interesting features that are not usually seen in adult patients. Above all, they showed the possible presence of acute network injury in the linked structures following the major brain insult in infants. The human brain is a complex system that is organized in an interconnected manner. Different brain regions communicate to efficiently process and integrate information [5]. This brain connectivity architecture is named the "neural network." By the time of normal birth, a greater part of the structural network architecture needed in normal brain function is built [6]. Until now, secondary changes following brain damage have not attracted as much attention as the primary insult. We particularly focused on this consequential event of neonatal cerebral infarction in this article. Multiple complex processes, such as glutamatergic excitotoxic injury caused by damaged cortical neurons or loss of the trophic support of neurons causing secondary degeneration, are said to give rise to network injuries [7]. Newborns and immature infants, usually younger than 1 year old, are particularly vulnerable to this type of injury due to scarcer somatodendritic architecture with less axonal collaterals [2].

Several investigators have reported acute network injury recognized in the corticospinal tract at the level of the posterior limb of the internal capsule, cerebral peduncles, basis pontis, and medullary pyramid [8] and others in the thalamus and corpus callosum [2]. Both of our cases confirmed this characteristic involvement found in previous studies with thorough pyramidal tract involvement from the level of the internal capsule through the lower medulla. What is more appealing about our cases is that the site of network injury not only followed longitudinal extensions of the neural tract but also proceeded along transverse connections that continued to reach the contralateral hemisphere. Case A had a main lesion at the right MCA territory, which is part of the anterior circulation region, and the secondary change was noticed on the anterior corpus callosum. Case B showed vast damage over the right MCA and posterior cerebral artery territories, which belong to both the anterior and posterior circulation regions. In this case, a subsidiary lesion was found at the splenium of the corpus callosum. These findings may account for that the site of network injury varies according to initial lesion locations; it does not necessarily remain in the same vascular territory and can project in an omnidirectional manner as long as the brain parts are interconnected as network system.

Besides, both of our cases presented the temporal changes of cerebral infarction as well as network injury seen in brain imaging of young patients. The sequential changes of diffusion images seem quite different between newborns and adults. The previous publication showed that perinatal ischemic stroke patients exhibited higher DWI signal intensity in the lesion than that in the contralateral asymptomatic brain until day 4 , then continued to decrease and reached equal by day 12 [4]. This process of pseudonormalization usually begins after 2 weeks in mature brains [9]. Case B demonstrated that the fully developed pseudonormalization of the main infarcted lesion and the subordinate pyramidal and splenial signal changes by day 15 , concurring with previous knowledge. Regarding the appearance of network injury, the literatures have presented hyperintensities detected within 7 or 8 days from symptom onset [2]. Case A confirmed this behavior with the newly signified secondary lesion at the anterior corpus callosum, internal capsule, and thalamus on day 7.

In fact, the study is subjected to several limitations. First of all, this research is merely a case study with limited representatives, and the findings may not be generalized. Also, it was

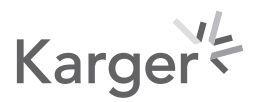


incapable of elucidating the pathomechanism or the exact causality of the phenomenon in this observational study. Nonetheless, the patients did not show clinical seizure any longer after admission, the possibility of seizure disorder causing signal alterations on the brain image cannot be certainly denied. Further research is warranted to reveal the undiscovered aspect of brain network injuries.

\section{Conclusion}

Acute network injury occurs secondarily after various brain insults. It appears after approximately 7 days from the onset of the disease in the part of the corticospinal tract, thalamus, or corpus callosum. Network injury may occur either in a longitudinal or transverse form. It appears as a high signal intensity lesion in DWI, mostly seen in neonates or young infants. Pseudonormalization of the network lesion completes around a week from its first appearance. As ominous neurological outcomes are thought to be related to acute network injuries, a better understanding of the phenomenon could be pivotal in improving diagnosis and management. Further systematic, large-sized studies are expected in the future.

\section{Statement of Ethics}

The studies involving human participants were reviewed and approved by the institutional review board of Keimyung University Dongsan Medical Center, South Korea (Institutional Review Board Number 2020-09-073). Written informed consent was obtained from the parents of patients for publication of this case report and any accompanying images.

\section{Conflict of Interest Statement}

The authors have no conflict of interest to declare.

\section{Funding Sources}

The authors did not receive any funding for this study.

\section{Author Contributions}

S.K. interpreted the data and wrote the first draft and revision of the manuscript. S.-I.S., H.P., and J.S.K. were involved in managing the patients, and reviewed and edited the manuscript. J.-H.H. was involved in managing the patients, designed and conceptualized the study, interpreted the data, and revised the manuscript.

\section{Data Availability Statement}

All data generated or analyzed during this study are included in this article. Further inquiries can be directed to the corresponding author. 


\section{References}

1 Fernández-López D, Natarajan N, Ashwal S, Vexler ZS. Mechanisms of perinatal arterial ischemic stroke. J Cereb Blood Flow Metab. 2014;34(6):921-32.

2 Okabe T, Aida N, Niwa T, Nozawa K, Shibasaki J, Osaka H. Early magnetic resonance detection of cortical necrosis and acute network injury associated with neonatal and infantile cerebral infarction. Pediatr Radiol. 2014;44(5):597-604.

3 Righini A, Doneda C, Parazzini C, Arrigoni F, Matta U, Triulzi F. Diffusion tensor imaging of early changes in corpus callosum after acute cerebral hemisphere lesions in newborns. Neuroradiology. 2010;52(11):102535.

4 Dudink J, Mercuri E, Al-Nakib L, Govaert P, Counsell SJ, Rutherford MA, et al. Evolution of unilateral perinatal arterial ischemic stroke on conventional and diffusion-weighted MR imaging. A J N R Am J Neuroradiol. 2009; 30(5):998-1004.

5 Sporns 0. Structure and function of complex brain networks. Dialogues Clin Neurosci. 2013;15(3):247-62.

6 Smyser CD, Wheelock MD, Limbrick DD Jr., Neil JJ. Neonatal brain injury and aberrant connectivity. Neuroimage. 2019;185:609-23.

7 Govaert P, Zingman A, Jung YH, Dudink J, Swarte R, Zecic A, et al. Network injury to pulvinar with neonatal arterial ischemic stroke. Neuroimage. 2008;39(4):1850-7.

8 Kirton A, Shroff M, Visvanathan T, deVeber G. Quantified corticospinal tract diffusion restriction predicts neonatal stroke outcome. Stroke. 2007;38(3):974-80.

9 Mader I, Schöning M, Klose U, Küker W. Neonatal cerebral infarction diagnosed by diffusion-weighted MRI: pseudonormalization occurs early. Stroke. 2002;33(4):1142-5. 